Research Article

\title{
Vibration Torque Suppression for Magnetically Suspended Flywheel Using Improved Synchronous Rotating Frame Transformation
}

\author{
Cong Peng $\left(\mathbb{D}\right.$, Kaiwen Cai ${ }^{(D}$, Zhiquan Deng $\mathbb{D}^{D}$, and Kexiang Li ${ }^{(D)}$ \\ College of Automation Engineering, Nanjing University of Aeronautics and Astronautics, Nanjing 211106, China \\ Correspondence should be addressed to Cong Peng; pengcong.2006@163.com
}

Received 8 January 2019; Accepted 16 April 2019; Published 12 May 2019

Academic Editor: Mario Terzo

Copyright (c) 2019 Cong Peng et al. This is an open access article distributed under the Creative Commons Attribution License, which permits unrestricted use, distribution, and reproduction in any medium, provided the original work is properly cited.

\begin{abstract}
Synchronous vibration, a common issue in active magnetic bearing (AMB) system, is mainly caused by mass imbalance of the rotor. It comes with high-power consumption and serious impact on the housing base, dramatically degrading the performance of AMB. Magnetically suspended flywheel (MSFW), which owns a flat rotor and consequently shows strong gyroscopic effects even at low operating speed, requires additional attention not only for suppressing the synchronous vibration but also for maintaining the overall stability faced with the coupled dynamics. In this work, in order to suppress the vibration torques in MSFW with significant gyroscopic effects, an improved synchronous rotating frame- (SRF-) based control method is proposed. The proposed method introduces the compensation phase for stability adjustment and aims at simultaneously suppressing the synchronous components in the coupled axes. Firstly, the vibration torque model of MSFW is established, and the baseline control strategy for suspension and gyroscopic effects restrain is derived. Then, the principle and implementation of the improved SRF-based vibration torque method are analyzed, which aims at suppressing the synchronous vibration torques through attenuating synchronous components in coil currents. Moreover, the stability of the overall closed-loop system is analyzed. Finally, the effectiveness of the proposed method is verified through simulation and experimental results.
\end{abstract}

\section{Introduction}

Magnetic bearings have seen great development in a wide range of industrial fields. Unlike conventional mechanical bearings, magnetic bearings utilize magnetic forces to support a shaft without any physical contact. It offers advantages of no friction, no lubrication, and long lifespan [1-5]. One of the most promising features of magnetic bearings is that the dynamic performance of the shaft is able to be shaped to different applications. These superior characteristics make magnetic bearings widely used in various fields, such as flywheels and high-speed motors [6-9].

Periodical disturbance is a common concern in magnetic bearings. It induces fluctuating current in the coil and further generates the vibration forces and torques [10-12]. A dominant source of the disturbance is the mass imbalance of the rotor. The mass imbalance leads to the misalignment of the principle axis of inertia and the axis of its geometry. It is unrealistic to achieve a mass-balanced rotor by sophisticated manufacturing; even though possible, mass shifts occur when the rotor rotates at high speed. To resolve the problem introduced by mass imbalance, adopting active control strategy for the active magnetic bearings is a superior and reliable option. Active vibration control strategy can force the rotor to rotate about its inertial axis, thus reducing the synchronous vibrations caused by mass imbalance. The active magnetic bearings usually support a rotor in 5 degrees of freedom (DOFs). A slim rotor, which has a small ratio of the moment of inertia, has such weak gyroscopic effects that the magnetic bearing system can be regarded as five singleDOF decoupled systems. Thus, the active control method can be implemented on every axis, and it is equivalent to five single-input single-output (SISO) systems. However, a flat 
rotor suspended by the magnetic bearings possesses the strong gyroscopic effects [13]. The gyroscopic effects lead to the coupling dynamics in the radial direction. It is especially serious for a magnetically suspended flywheel (MSFW). Flywheel is an essential part installed onboard satellites, whose vibration is a threat to the performance of the satellite payload. Consequently, the control strategy for MSFW is confronted with a multiple-input multiple-output (MIMO) system. The active synchronous vibration control for the coupled MIMO system with serious gyroscopic effects has been a challenging control problem in the flat AMB-rotor system.

Objectives of vibration suppression control fall into two categories: (1) imbalance compensation and (2) automatic balancing [14]. Imbalance compensation strategies force the rotor rotate around its geometric axis to achieve high rotational precision. In contrast, automatic balancing strategy makes the rotor rotate around its inertial axis, in which case the vibration forces/torques are eliminated. To achieve automatic balancing, there exists many control schemes. A generalized notch filter was proposed in [15]; it adopted a transfer matrix to adjust the phase of compensation signal and assured a wide stable speed range. The effectiveness of the notch filter method relied on the loop frequency response, which could be easily measured in practice. Based on the principle of notch filter, the transfer matrix was modified in [16] with a leading phase compensator, making it easier to regulate the stability of the closed-loop system. An adaptive feedback method was developed in [17]. In [17], the synchronous compensation signal was generated automatically to make the controller not respond to the synchronous displacement signal. This method was effective regardless of the loop frequency response. An LMS adaptive filter [18] was proposed to achieve imbalance compensation in a hard-disk system. Compared to the notch filter method, LMS adaptive filter offered the advantage of tuning parameters directly. Extensive studies of vibration control such as automatic learning algorithms and robust controller can be found in [19-22].

However, these aforementioned methods are only feasible for the SISO system, where the controlled plant is decentralized. Moreover, the stability problem caused by gyroscopic effects is not taken into consideration. To attenuate vibration torques in a MIMO system, a crossfeedback notch filter was proposed in [23]. The vibration torque suppression was obtained by a cross-feedback notch filter, and the gyroscopic effects were reduced by a crossfeedback controller. The stability was maintained and the effectiveness was verified through analysis and experiments. However, its controller order is large, which increased the computational burden in digital signal processor. Eliminating vibrations as well as overall stability preservation for a MIMO system is of significance. There remain much improvements for applying conventional control schemes.

The idea of synchronous rotating frame (SRF) method has been widely used in the fields of power electronic and motor control to extract and track signals [24-29]. In [24], the SRFbased strategy was utilized to obtain a phase locked loop (PLL), which consumed less computational resources. A paralleled current controller structure for three-phase grid converter was proposed in [27]; it deployed the controller in rotating frame and performed reduction at multiple harmonics. In [29], to simplify the calculation of the real and reactive power quantities, rotating frames reference was selected to clearly represent the positive and negative sequence voltages and currents. Nevertheless, in magnetic bearing applications, there is little work of using the SRF-based method in the MIMO system with regard to vibration suppression. Compared to the existing strategies which aimed at eliminating the synchronous signals in magnetic bearing system, the SRF-based method offers advantages of simplified analysis on closed-loop system stability as well as light pressure on computation for digital signal processor [30].

This work presents an improved SRF-based method to suppress vibration torques in the MSFW. First, the dynamic model for the MSFW in coupled MIMO radial directions is analyzed and the model for synchronous vibration torques is established. Then, the strategy of using the improved SRFbased method is proposed to suppress the synchronous vibration torques. The improved SRF not only adopts compensation phase for closed-loop stability adjustment but also attenuates the vibration torques in the coupled axes due to the rotor gyroscopic effects. Simulation and experimental results prove that the application of improved SRF-based method in the MIMO system is effective in suppressing vibration torques as well as maintaining the overall system stability.

This paper is organized as follows. The synchronous vibration torques and MIMO dynamics in the radial directions of the MSFW system are analyzed in Section 2. In Section 3, the improved SRF-based synchronous vibration control method is proposed for the vibration torque suppression for the MIMO coupled system. Simulation and experimental results for vibration torques are presented in Section 4. Finally, conclusions are given in Section 5.

\section{MSFW Dynamic Model}

Figure 1 shows the configuration of MSFW. The MSFW in this work is supported by active magnetic bearings in 5 DOFs, of which the 2 DOFs of radial translational motion are under the control of radial bearings, and the 3-DOF-included axial translational motion and radial rotational motion are under the control of axial bearings. The novel specific structure for the MSFW has the advantages in reducing the axial length and providing a better controllability. This work focuses on the control of the radial rotational motion. More details on the magnetic bearing structure can be found in [31].

Denote the geometric center of the rotor as $C_{\mathrm{g}}$ and the mass center as $C_{i} . x, y$, and $z$ axes construct the generalized coordinate system with the mass center as the origin. $\alpha$ and $\beta$ refer to the rotational angle of the rotor about $x$ - and $y$-axis. It is noted that the mass imbalance is composed of the static imbalance and the dynamic imbalance. For the radial rotational motion, the misalignment of the principle axis and the geometric axis of the rotor lead to the dynamic imbalance and further generate the vibration torques. The vibration torques in the radial directions can be presented as 


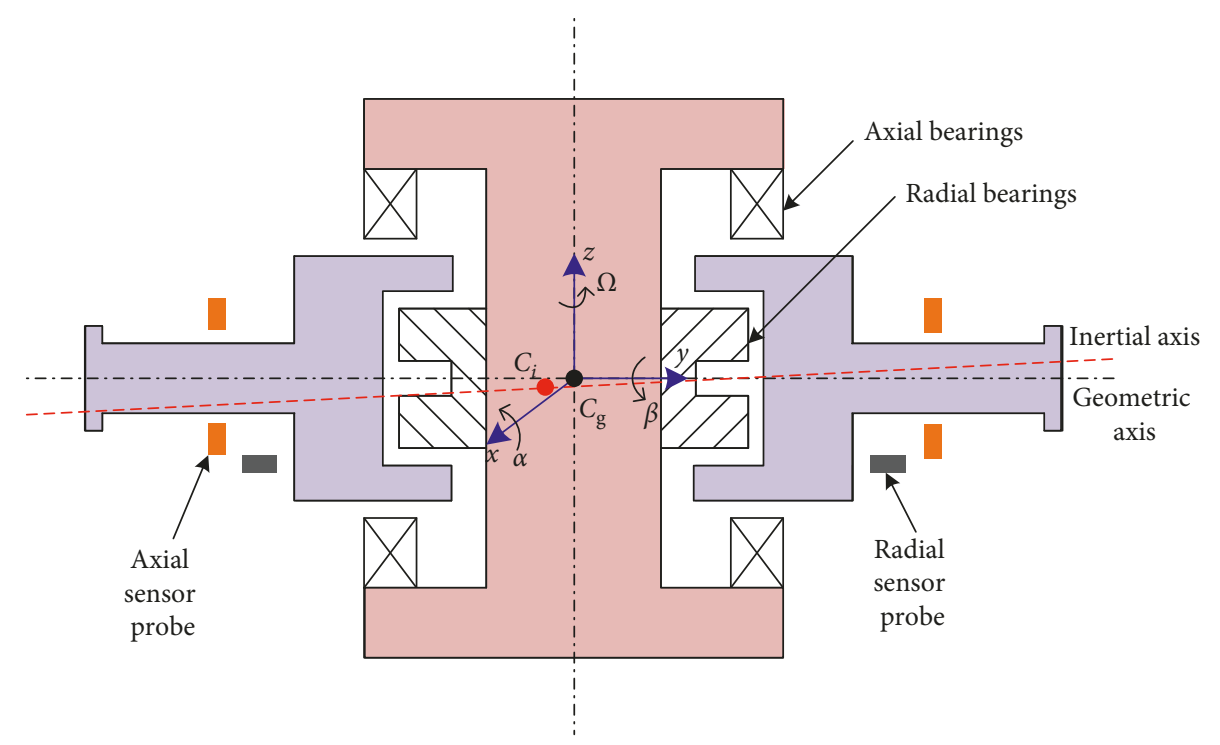

FIgURE 1: The schematic diagram of the MSFW.

$$
\begin{aligned}
& T_{d x}=\left(J_{x}-J_{z}\right) \lambda_{d} \Omega^{2} \cos \left(\Omega t+\phi_{d}\right), \\
& T_{d y}=\left(J_{y}-J_{z}\right) \lambda_{d} \Omega^{2} \sin \left(\Omega t+\phi_{d}\right),
\end{aligned}
$$

where $J_{x}, J_{y}$, and $J_{z}$ are the moment of inertia with respect to $x$-, $y$-, and $z$-axis and $\lambda_{d}$ and $\phi_{d}$ are the amplitude and initial phase of the dynamic imbalance, respectively.

Then, according to Newton's law of motion, the dynamical model of the rotor-AMB system in the radial directions accompanied with the dynamic imbalance [32] can be expressed as

$$
\begin{aligned}
& J_{x} \ddot{\alpha}-J_{z} \dot{\beta} \Omega=k_{i z} r_{\mathrm{m}} i_{\beta}+2 k_{s z} r_{\mathrm{m}}^{2} \beta+m_{d} \lambda_{d} \Omega^{2} \cos \left(\Omega t+\phi_{d}\right), \\
& J_{y} \ddot{\beta}+J_{z} \dot{\alpha} \Omega=k_{i z} r_{\mathrm{m}} i_{\alpha}+2 k_{s z} r_{\mathrm{m}}^{2} \alpha+m_{d} \lambda_{d} \Omega^{2} \sin \left(\Omega t+\phi_{d}\right),
\end{aligned}
$$

where $i_{\alpha}$ and $i_{\beta}$ are the control currents in the radial directions and $k_{i z}$ and $k_{s z}$ are the current stiffness and the displacement stiffness, respectively.

Figure 2 shows the complete baseline control diagram for the radial rotational motion of the rotor. The complete dynamical model of the radial rotational motion, which includes the dynamics, the baseline suspension controller, the power amplifier, and the sensor, is presented as

$$
\begin{aligned}
J_{y} \ddot{\beta}(t)+J_{z} \Omega \dot{\alpha}(t)-2 k_{s z} r_{\mathrm{m}}^{2} \beta(t)= & 2 k_{i z} r_{\mathrm{m}} g_{\mathrm{a}} g_{s} \\
& \cdot\left[g_{\mathrm{b}} \beta(t)+g_{\mathrm{cr}} \Omega \alpha(t)\right]+T_{d y},
\end{aligned}
$$

$$
\begin{aligned}
J_{x} \ddot{\alpha}(t)-J_{z} \Omega \dot{\beta}(t)-2 k_{s z} r_{\mathrm{m}}^{2} \alpha(t)= & 2 k_{i z} r_{\mathrm{m}} g_{\mathrm{a}} g_{s} \\
& \cdot\left[g_{\mathrm{b}} \alpha(t)-g_{\mathrm{cr}} \Omega \beta(t)\right]+T_{d x}
\end{aligned}
$$

where $r_{\mathrm{m}}$ is the distance from the geometric center to the axial sensor, $g_{\mathrm{b}}(s)$ denotes the baseline PID controller for rotor suspension, $g_{\mathrm{cr}}(s)$ denotes cross-feedback controller aimed at

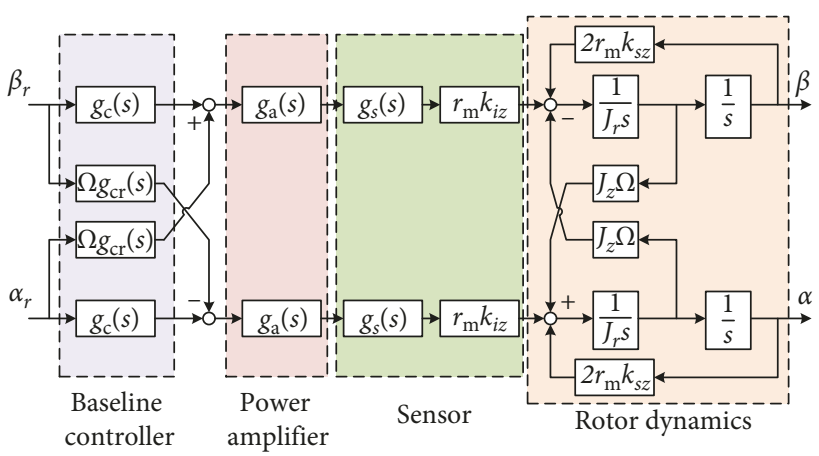

FIgURE 2: The baseline control diagram for the radial rotational motion of the rotor, including the baseline suspension controller, the power amplifier, the sensor system, and the rotor dynamics.

attenuating gyroscopic effects, $g_{\mathrm{a}}(s)$ and $g_{s}(s)$ denote power amplifier and current sensor, respectively, and $T_{d x}$ and $T_{d y}$ are the vibration torques for $\alpha$ and $\beta$ directions, respectively. According to equations (3) and (4), it is noted that the radial rotational system is a multi-input multi-output (MIMO) system and coupled with the rotational speed.

In order to simplify the stability analysis of the coupled MIMO system, we reconstruct the coupled system into a complex SISO system. Note that in the generalized coordinate, $\alpha$ leads $\beta$ by $90^{\circ}$. Thus, a plural variable can be defined as

$$
\eta(t)=\beta(t)+j \alpha(t),
$$

where $j$ is plural unit 1 , that is, $j^{2}=-1$. Multiplying equation (3) by $j$ and then adding the result to equation (6) yields the differential equation as

$$
\begin{aligned}
J_{r} s^{2} \eta(s)-j J_{z} \Omega s \eta(s)-2 k_{s z} r_{\mathrm{m}}^{2} \eta(s)= & 2 r_{\mathrm{m}} k_{i z} g_{\mathrm{a}}(s) g_{s}(s) \\
& \cdot\left[g_{\mathrm{b}}(s)+j g_{\mathrm{cr}}(s)\right] \eta_{r}(s) .
\end{aligned}
$$


According to equation (6), the equivalent SISO system corresponds to a negative feedback control unit, which is shown in Figure 3.

As presented in Figure 3, $G(s)$ is the equivalent controller and $H(s)$ represents the equivalent control plant. $G(s)$ and $H(s)$ are derived as

$$
\begin{aligned}
& G(s)=g_{\mathrm{a}}(s)\left[g_{\mathrm{c}}(s)+j g_{\mathrm{cr}}(s)\right], \\
& H(s)=\frac{2 r_{\mathrm{m}} k_{i z} g_{s}(s)}{J_{\mathrm{r}} s^{2}-j J_{z} \Omega s-2 k_{s z} r_{\mathrm{m}}^{2}} .
\end{aligned}
$$

Thus, the complicated MIMO system for MSFW in radial rotational direction is converted to an equivalent SISO system, making it easy for us to do stability analysis as well as implement vibration suppress algorithm. It is worthy to note that the signals in the equivalent SISO system are plural ones.

\section{Improved SRF for Vibration Torque Suppression}

3.1. Theory Analysis of SRF. The basic idea of SRF is shown in Figure 4, where $C x_{s} y_{s}$ is the stationary reference frame, $C x_{r} y_{r}$ is the synchronous rotating reference frame, $\eta(t)=\beta(t)+j \alpha(t)$ is the space vector of specific frequency, which rotates about the origin at the speed of $k \Omega, k$ is the harmonic order, and $\Omega$ is the rotor angular speed. If we focus on the synchronous component in radial rotational motion, then in this case $k=1$. In the reference frame $C x_{s} y_{s}$, $\eta(t)$ rotates about the origin at speed of $\Omega$. When the reference frame $C x_{r} y_{r}$ spindles about the origin at the same speed, $\eta(t)$ becomes a stationary vector in the rotational reference frame $C x_{r} y_{r}$. The frequency of $\eta(t)$ is adjustable when the rotational speed of its reference frame is under control.

There are two kinds of SRF transformation, the positive transformation and the negative transformation [26]. Positive transformation converts the frequency of specific component in input signal to zero, while negative transformation converts it back to origin. Both positive transformation and negative transformation only convert the frequencies of signals, while the amplitude of signals is preserved. $T_{\mathrm{P}}(k \Omega)$ and $T_{\mathrm{N}}(k \Omega)$ are the positive transformation matrix and the negative transformation matrix, respectively. Then, the two types of transformation matrices are derived as

$$
\begin{aligned}
& T_{\mathrm{P}}(k \Omega)=\left[\begin{array}{cc}
\cos \left(k \Omega t+\theta_{k}\right) & \sin \left(k \Omega t+\theta_{k}\right) \\
-\sin \left(k \Omega t+\theta_{k}\right) & \cos \left(k \Omega t+\theta_{k}\right)
\end{array}\right], \\
& T_{\mathrm{N}}(k \Omega)=\left[\begin{array}{cc}
\cos (k \Omega t) & -\sin (k \Omega t) \\
\sin (k \Omega t) & \cos (k \Omega t)
\end{array}\right],
\end{aligned}
$$

where $\theta_{k}$ is the initial phase of transformation. Thus, the positive transformation can be presented as

$$
\eta_{\mathrm{o}}(t)=T_{\mathrm{P}}(k \Omega) \cdot \eta_{\mathrm{i}}(t)
$$

while negative transformation can be presented as

$$
\eta_{\mathrm{o}}(t)=T_{\mathrm{N}}(k \Omega) \cdot \eta_{\mathrm{i}}(t)
$$

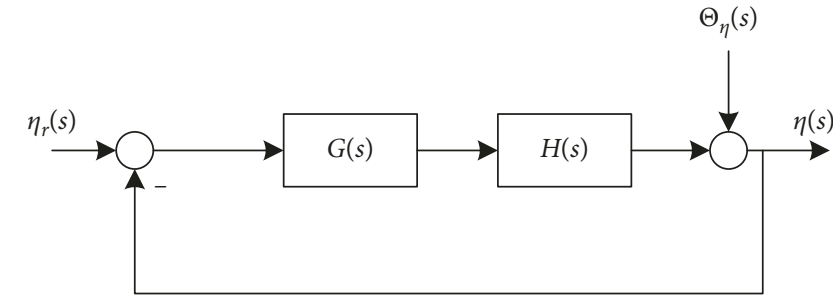

FIgURE 3: The equivalent SISO negative feedback control unit. G(s) is the equivalent controller and $H(\mathrm{~s})$ is the equivalent control plant.

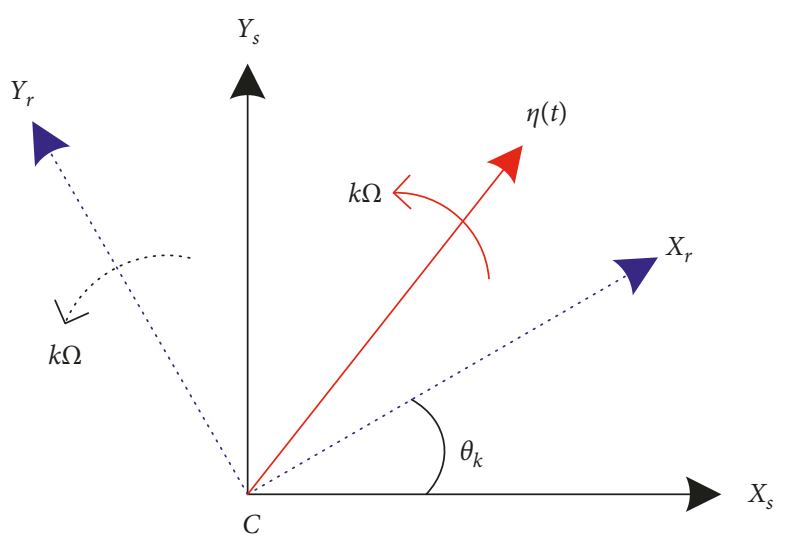

Figure 4: The basic idea of SRF.

where $\eta_{\mathrm{i}}(t)$ is the input signal before transformation and $\eta_{\mathrm{o}}(t)$ is the output signal after transformation.

It is noted that the signal that the positive and negative transformation implemented has multiple frequencies. By using positive transformation, the frequency of the component which is synchronous to the rotational speed is converted to zero and the frequencies of the other components remain nonzero constant. While after negative transformation, the frequency of all components in the signal converts back to its original value.

3.2. Strategy of the SRF-Based Vibration Suppression Method. Figure 5 shows the baseline structure for vibration suppression based on SRF. $\eta_{[.]}(t)$ is the angle in generalized coordinate. Specifically, $\eta_{\mathrm{in}}(t)$ is the original signal. $\eta_{\mathrm{dc}}(t)$ is the signal after positive transformation; $\hat{\eta}_{\mathrm{dc}}(t)$ is the signal filtered by low-pass filter, which is also the input signal into the negative transformation. $\eta_{\text {out }}(t)$ is the signal after negative transformation.

According to equations (9) and (10), the positive transformation and negative transformation in Figure 5 are derived as

$$
\begin{aligned}
& \eta_{\mathrm{dc}}(t)=T_{\mathrm{P}}(k \Omega) \cdot \eta_{\text {in }}(t), \\
& \eta_{\text {out }}(t)=T_{\mathrm{N}}(k \Omega) \cdot \widehat{\eta}_{\mathrm{dc}}(t) .
\end{aligned}
$$

Positive transformation indicates that the frequency of all components in the signal decreases by $k \Omega$, and negative transformation means that the frequency of the harmonic 


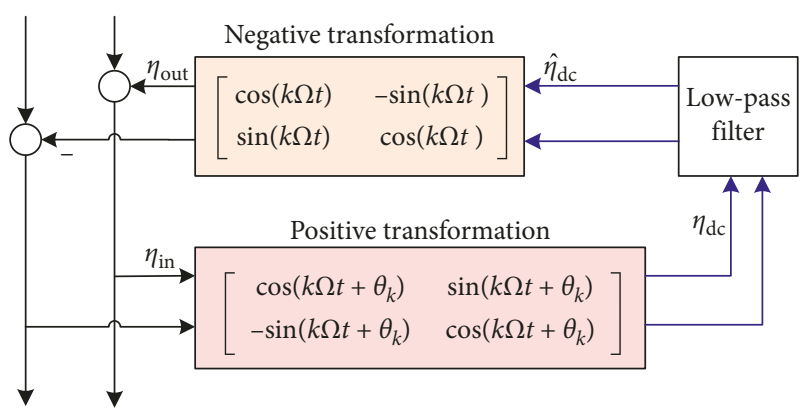

Figure 5: The strategy of the SRF-based method for vibration suppression.

increases by $k \Omega$. Thus, equations (11) and (12) can be represented by using the exponential expression as

$$
\begin{aligned}
& \eta_{\mathrm{dc}}(t)=\eta_{\text {in }}(t) e^{-j\left(k \Omega t+\theta_{k}\right)}, \\
& \eta_{\text {out }}(t)=\widehat{\eta}_{\mathrm{dc}}(t) e^{j k \Omega t} .
\end{aligned}
$$

Applying Laplace transformation to equations (13) and (14) results in

$$
\begin{aligned}
\eta_{\mathrm{dc}}(s) & =\eta_{\text {in }}(s+j k \Omega) e^{-j \theta_{k}}, \\
\eta_{\text {out }}(s) & =\widehat{\eta}_{\mathrm{dc}}(s-j k \Omega) .
\end{aligned}
$$

Then, the signal that passes low-pass filter is presented as

$$
\widehat{\eta}_{\mathrm{dc}}(s)=\eta_{\mathrm{dc}}(s) H_{\mathrm{p}}(s),
$$

where $H_{\mathrm{p}}(s)$ is the low-pass filter and can have the typical structure as

$$
H_{\mathrm{p}}(s)=\frac{1}{\tau s+1}
$$

where $\tau$ is the time constant of the filter. Furthermore, as presented in Figure 4, the open-loop transfer function of SRF-based suppression method is concluded as

$$
G_{k}(s)=\frac{\eta_{\text {out }}(s)}{\eta_{\text {in }}(s)}=\frac{e^{-j \theta_{k}}}{\tau(s-j k \Omega)+1} .
$$

Hence, the closed-loop transfer function of SRF is obtained as

$$
N_{k}(s)=\frac{1}{1+\kappa G_{k}(s)}=\frac{\tau(s-j k \Omega)+1}{\tau(s-j k \Omega)+1+\kappa e^{-j \theta_{k}}},
$$

where $\kappa$ is the gain of the SRF transformation loop and $\theta_{k}$ is used as the compensation phase to adjust the closed-loop system stability.

3.3. Design of Low-Pass Filter. A first-order low-pass filter is adopted inside the SRF-based vibration suppression strategy, as presented in equation (17). Time constant $\tau$ decides the speed of convergence and the cutoff frequency of the filter. The time constant can be calculated as

$$
\tau=\frac{1}{2 \pi f_{c}}
$$

where $f_{\mathrm{c}}$ denotes the cutoff frequency.
When passing through the low-pass filter, the signal whose frequency is below $f_{c}$ retains its amplitude, while the amplitude of the signal whose frequency is beyond $f_{\mathrm{c}}$ is suppressed. Then, the characteristic of the low-pass filter can be presented as

$$
H_{\mathrm{p}}(j \omega)=\frac{1}{1+j \omega \tau}=\frac{1}{1+j\left(\left(f-f_{0}\right) / f_{\mathrm{c}}\right)},
$$

where $f_{0}$ is the frequency of the rotational speed and $f$ denotes the frequency of the vibration signal. After positive transformation, the frequency of the vibration disturbance signal is $f-f_{0}$.

The vibration signal can be regarded as the combination of multiple sinusoidal disturbance signals, whose frequencies are $f_{1}, f_{2}, \ldots, f_{n}$, respectively, and the frequency of the synchronous vibration signal is $f_{i}=f\left(f_{i} \in f_{1}, f_{2}, \ldots, f_{n}\right)$. When the synchronous vibration signal passes through the lowpass filter, the specific frequency can be substituted into equation (21) and obtained as

$$
\left.H_{\mathrm{p}}(j \omega)\right|_{f=f_{0}}=1 .
$$

It indicates that the synchronous vibration signal passes through the low-pass filter without losing its amplitude. For $f_{i} \neq f\left(f_{i} \in f_{1}, f_{2}, \ldots, f_{n}\right)$, there are two factors that decide the reduction of the amplitude of a signal, $f$ and $f_{\mathrm{c}}$. According to equation (21), it can be observed that the larger the $\left|f-f_{0}\right|$ is obtained, the larger the amplitude is reduced. To obtain the synchronous sinusoidal vibration signal, this property is exactly what is desired.

As the frequency of the signal that is designed to pass the filter is zero, one could set the cutoff frequency $f_{\mathrm{c}}$ as small as possible, in which case a narrow-band notch filter can be obtained. Nevertheless, a small cutoff frequency means narrow bandwidth, resulting in slow convergence speed. On the other hand, if $f_{\mathrm{c}}$ is a large value, then more high-frequency signals would pass the low-pass filter; this means the band of the notch filter is broader. A broader band notch filter brings instability to the controller. Thus, there should be a trade-off between the convergence speed and the stability of the filter.

3.4. Analysis of System Stability. Figure 6 shows the whole control diagram with the SRF plugged into the baseline MSR-rotor control system. This structure has the merits of independent tuning of the nominal position controller and the proposed vibration controller. The sensitivity function of the inner loop $[G, H]$ is presented as

$$
S(s)=[1+G(s) H(s)]^{-1} .
$$

To evaluate the stability of the system, it is necessary to investigate the poles and zeros of the closed loop. The characteristic polynomial of the closed-loop $\left[G, H, G_{k}\right]$ is

$$
\Delta=1+S(s) G_{k}(s) \text {. }
$$

The system is stable only if all zeros of the characteristic polynomial are located in the left-hand side of the complex plane (LHP). Generally, we assume the system denoted by 


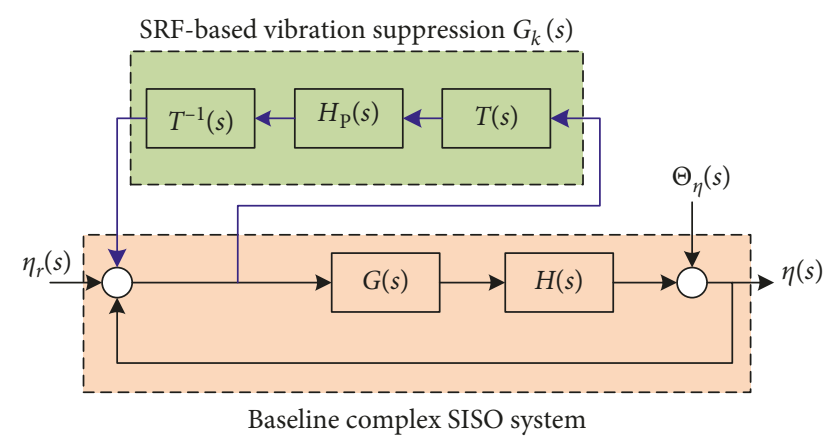

FIgURE 6: The whole control diagram for synchronous vibration suppression with the embedded SRF method.

$S(s)$ is stable, which means the poles of $S(s)$ are located in the LHP. The task is to determine the location of new poles imported by $G_{k}(s)$. Let $\Delta=0$, that is,

$$
\kappa \cdot e^{-j \theta_{k}} S(s)+\tau \cdot(s-j k \Omega)+1=0 .
$$

If $\kappa=0$, one of the roots of the characteristic polynomial is $s=-(1 / \tau)+j k \Omega$. Generally, $\tau$ is a constant number, which means the root is located within the field of $s=j k \Omega$. The root locus $s(\kappa)$ is a differentiable function at $\kappa=0$. Linearizing the root locus branch starting at $j k \Omega$ for $\kappa=0$ yields

$$
\left.\frac{d s(\kappa)}{d \kappa}\right|_{\kappa=0, s=j k \Omega}=-\frac{e^{-j \theta_{k}} S(j k \Omega)}{\tau} .
$$

Thus, there exists a small $\kappa$ such that the root locus branching from $s=j k \Omega$ stays in the LHP. The maximum damping can be achieved when the root locus derivatives given by equation (26) equal to $\pi$. Furthermore, the maximum damping means that the best stability of the closedloop system is obtained, that is,

$$
\left\{\begin{array}{l}
\operatorname{Re}\left[-\frac{e^{-j \theta_{k} S(j k \Omega)}}{\tau}\right]<0, \\
\operatorname{Im}\left[-\frac{e^{-j \theta_{k}} S(j k \Omega)}{\tau}\right]=0,
\end{array}\right.
$$

where $\operatorname{Re}[\cdot]$ means taking the real part and $\operatorname{Im}[\cdot]$ means taking the imaginary part. Actually, the insertion of $N_{k}(s)$ is supposed to reduce the bearing stiffness with respect to a narrow band of frequency at $k \Omega$, which means the bearing stiffness remains unaffected at any other frequencies.

\section{Simulation and Experimental Results}

4.1. Simulation and Experimental Setup. In order to evaluate the proposed synchronous vibration suppression scheme, a MSFW, as shown in Figure 7, is used to test the proposed $\mathrm{SRF}$ method performance on the vibration reduction. It also shows the measurement system for directly tested vibration torques of the MSFW. The experimental setup is composed of an interface table, a Kistler table 9253B12, and a signal processing unit. The Interface table is used to provide the rigid contact between the MSFW and the Kistler table. The
MSFW is mounted on the interface table. The Kistler table is the key instrument for the dynamic and quasistatic measurement of the three orthogonal components of force/ torque. The average value of the background noise is $100-200 \mathrm{mN}$, which is suitable for testing the microvibrations produced by MSFW. The signal processing unit includes the multichannel charge amplifier 5080 and the real-time multianalyzer. The real-time multianalyzer is used to process the microvibration data in real time, e.g., transforming the data in time domain into the frequency domain to distinguish the frequency contents. The parameters for the MSFW model are shown in Table 1. Without losing accuracy, the parameters are set in accordance with the actual equipment. The task of simulation and experiment is to verify that in the presence of the proposed method, the stability of the overall closed-loop system is preserved and the vibration torques are reduced.

4.2. Simulation Results. The task of parameters tuning of the proposed vibration controller is to locate the additional poles in the left half plane (LHP), such that the closed-loop system with the proposed vibration controller is stable. The original closed-loop system is a high-order system; in the absence of the proposed vibration controller, the original closed loop is stable with appropriate controller parameters, which means all poles of the original system are located in the LHP. The constant speed $1500 \mathrm{rpm}$ is chosen as typical low-speed range to analyze the stability performance. For the operating conditions, the bandwidth of the low-pass filter is chosen as $f_{c}=2.3 \mathrm{~Hz}$. It is noted that the choices of control gain $\kappa$ and $\theta_{k}$ depend on the operating speed of the rotor and the sensitivity function of the system. Large $\kappa$ will damage the closed-loop stability while small $\kappa$ will reduce the effects of vibration suppression.

Firstly, the compensation phase $\theta_{k}$ which used to stabilize the closed-loop system is discussed. Since the range of $\theta_{k}$ can be determined once $\kappa$ is settled, we use root locus to analysis the range of $\theta_{k}$. Before further discussion about $\kappa$, we may as well fix $\kappa$ at 100 . Figure 8(a) shows the root locus of the additional pole introduced by the proposed vibration controller when increasing the value of $\theta_{k}$ from $-180^{\circ}$ to $180^{\circ}$. As shown in Figure 8(a), there are four tracks of the root locus, which are denoted with 1, 2, 3, and 4. The pole starts from track 1 , then crosses the imaginary axis when $\theta_{k}=-100^{\circ}$, and enters track 2 and 3 . In track 4 , it stays in the LHP until $\theta_{k}$ reaches $50^{\circ}$. The poles that are located in the LHP indicate the closed loop is stable; thus, we get the range of $\theta_{k}$ that can stabilize the system is $\left(-100^{\circ}, 50^{\circ}\right)$.

Although all $\theta_{k}$ within the specific range is able to stabilize the system, the task to find an optimal value of $\theta_{k}$ is still of our interest. Figure 8 (b) shows the different root locus with the fixed $\theta_{k}$ and the varying gain $\kappa \in(10,300)$. For the simple description, the angle between the root locus at starting point and the real axis is denoted as the departure angle. According to Figure $8(\mathrm{~b})$, when $\theta_{k}=20^{\circ}$, the departure angle of the root locus is about $100^{\circ}$. While the departure angle becomes $150^{\circ}$ and $180^{\circ}$ when $\theta_{k}=0^{\circ}$ and $\theta_{k}=-20^{\circ}$, respectively. It can be seen that $\theta_{k}$ can adjust the departure 


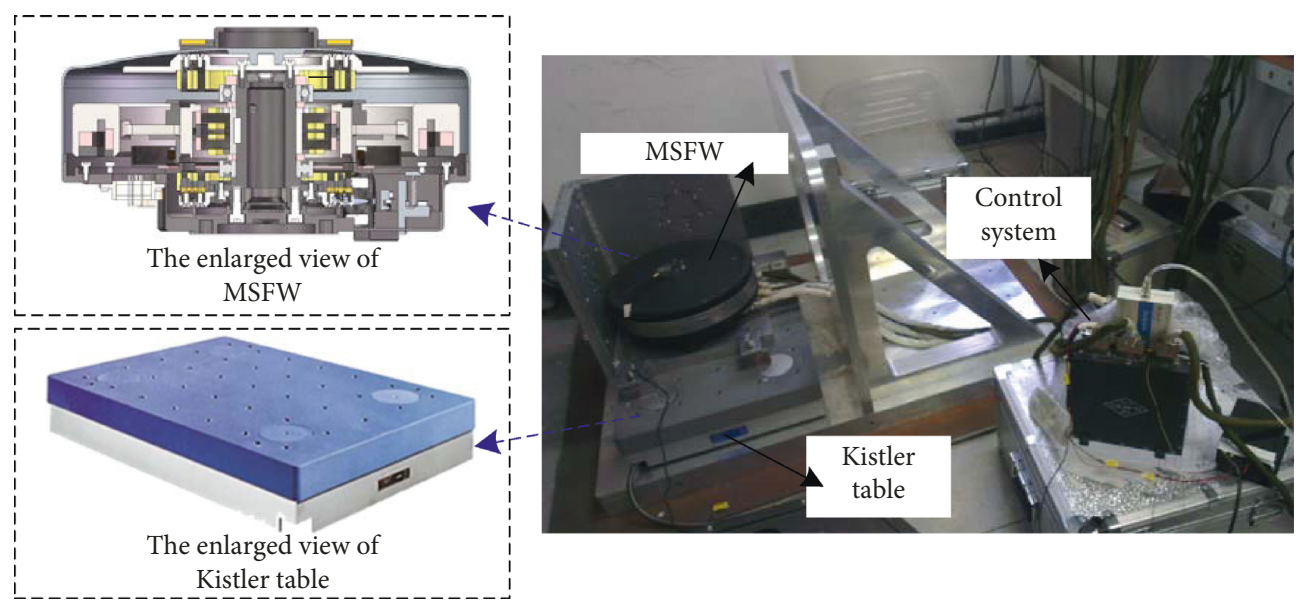

Figure 7: The experimental setup, including the tested MSFW, the Kistler table for vibration torques measurement, and control system for the algorithm implementation.

TABLE 1: Simulation model parameters.

\begin{tabular}{lc}
\hline Parameter & Value \\
\hline$m$ & $4.08 \mathrm{~kg}$ \\
$J_{x}, J_{y}$ & $0.015 \mathrm{~kg} \cdot \mathrm{m}^{2}$ \\
$J_{z}$ & $0.028 \mathrm{~kg} \cdot \mathrm{m}^{2}$ \\
$k_{s z}$ & $3.3 \times 10^{5} \mathrm{~N} / \mathrm{m}$ \\
$k_{i z}$ & $32 \mathrm{~N} / \mathrm{A}$ \\
\hline
\end{tabular}

angle of the root locus introduced by the proposed vibration controller. If $\theta_{k}$ is closer to the angle of $S\left(j \Omega_{0}\right)$, the departure angle of the root locus is closer to $180^{\circ}$. The departure angle of the root locus is associated with the damping of the system, and the optimal damping can be achieved when the departure angle is $180^{\circ}$. The result is in accordance with equation (27). According to the constraint given by equation (27), we can choose $\theta_{k}=-22^{\circ}$ for low operating speed condition to obtain an optimal damping. Without loss of generality, we choose $\theta_{k}=-11^{\circ}$ for high operating speed condition.

Furthermore, the control gain $\kappa$ and the compensation phase $\theta_{k}$ in the SRF loop are analyzed. Figure 9 shows the ratio of the amplitude of coil current with the proposed vibration controller to that without the proposed vibration controller. It is observed that when the closed loop is stable, different values of $\theta_{k}$ will not affect the performance of the proposed vibration controller in view of its performance in suppressing synchronous currents. Besides, it can be seen from Figure 9 that when $\kappa$ increases from 0 to 300, the ratio decreases from 1.0 to 0.03 ; that is, a larger $\kappa$ promises a better vibration suppression effect. On the other hand, where the larger $\kappa$ is chosen, the closer the pole is close to the imaginary axis. Hence, the large $\kappa$ would deteriorate the closed-loop stability. Thus, there should be a trade-off when choosing $\kappa$. Figure 9 shows that as the current reduction grows slowly after $\kappa$ reaches 100 , it is reasonable to choose $\kappa=100$ in this work.

Figures 10 and 11 show the simulation results of coil currents at low operating speed and high operating speed with different choices of $\kappa$ and $\theta_{k}$. As observed from Figures $10(\mathrm{a})-10(\mathrm{c})$, at low operating speed $1500 \mathrm{rpm}$, the amplitude of coil currents decreases when the proposed vibration controller is activated. Comparing Figure 10(a) with Figure 10(c), it is proved that a larger $\kappa$ contributes to a larger reduction of the amplitude of coil current. However, when different values of $\theta_{k}$ are chosen, it shows little difference with respect to the reduction of the amplitude of coil current. Moreover, the baseline controller will not be stable if the value of $\theta_{k}$ is inappropriate, which is shown in Figures $10(\mathrm{~d})$ and $11(\mathrm{~d})$. It makes sense that the compensation phase $\theta_{k}$ determines the stability range, not the suppression performance of the proposed vibration controller. Figure 11 shows that the proposed vibration controller is also effective at high operating speed $4800 \mathrm{rpm}$, where the amplitude of coil currents declines with the presence of the proposed vibration controller.

4.3. Experimental Results. To investigate the performance of the proposed synchronous vibration suppression, the vibration torques under constant rotational speed and varied rotational speed are considered. Figure 12 shows the vibration torque signal spectrums with and without the proposed vibration suppression strategy when the MSFW rotates at $1500 \mathrm{r} / \mathrm{min}$. Figure 13 shows the vibration torque signal spectrums with and without the proposed vibration suppression strategy when the MSFW rotates at $4800 \mathrm{r} / \mathrm{min}$. As can be observed from Figure 12(a), the maximum synchronous component of the vibration torques is $0.3 \mathrm{Nm}$ without suppression, while it reduces to less than $0.1 \mathrm{Nm}$ with the proposed method as shown in Figure 12(b). It can be deduced that there is greater than $67 \%$ reduction in the synchronous component of the vibration torque. The similar performance can be observed in Figure 13. The maximum peak-to-peak value of synchronous vibration torque is approximately $2.6 \mathrm{Nm}$ without any suppression. When the proposed method is implemented on the baseline control system, the maximum synchronous component reduces to $0.8 \mathrm{Nm}$. The experimental results for the vibration torque under the constant rotational speed indicate that the synchronous component can be greatly eliminated with the proposed SRFbased strategy. 


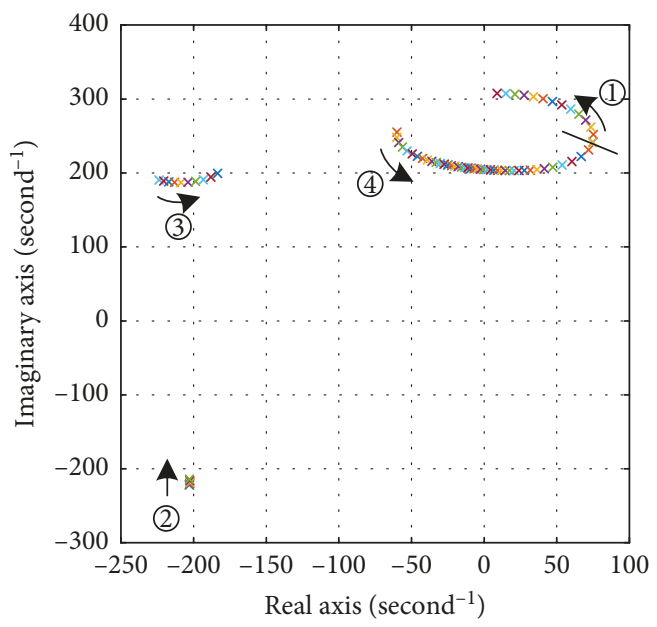

(a)

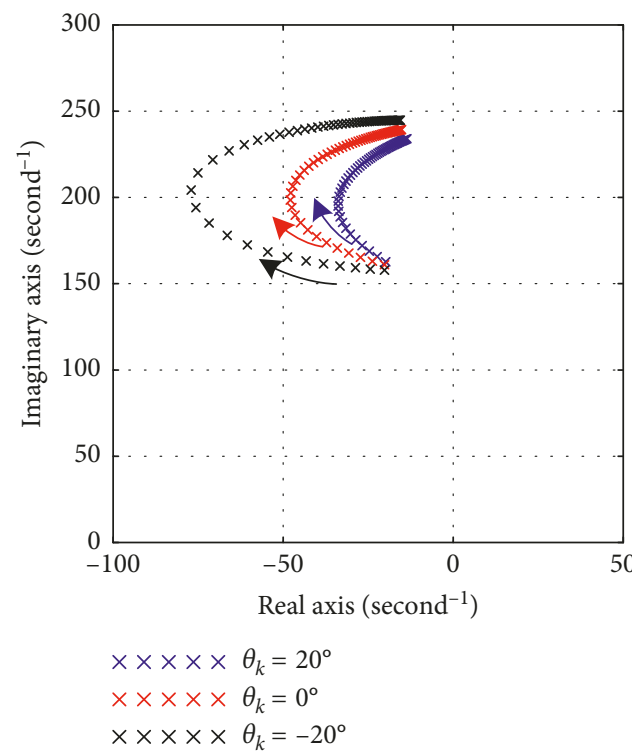

(b)

Figure 8: The locus of additional pole introduced by the proposed SRF. (a) $\theta_{k}$ varies from $-180^{\circ}$ to $180^{\circ}$. (b) $\kappa$ varies from 10 to 300 .

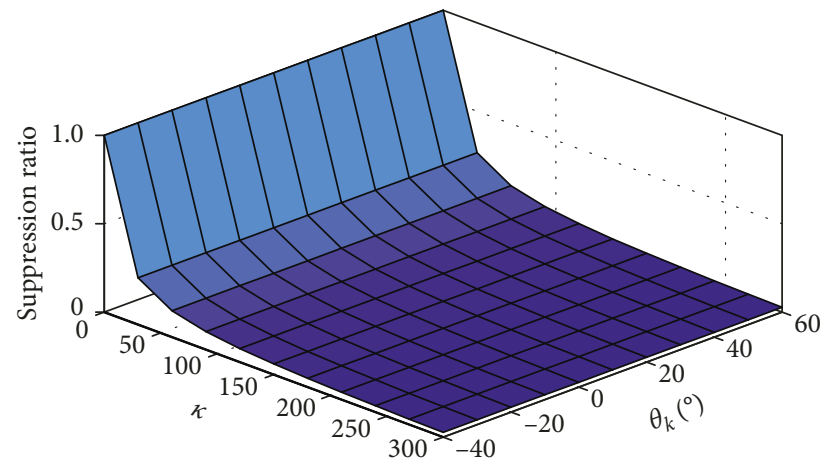

Figure 9: Simulation results of coil current ratio with different choices of $\kappa$ and $\theta_{k}$ ( $\alpha$ direction).

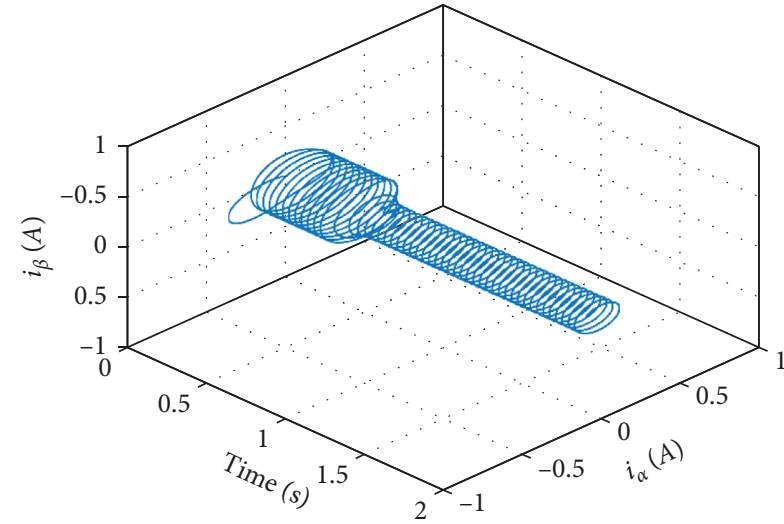

(a)

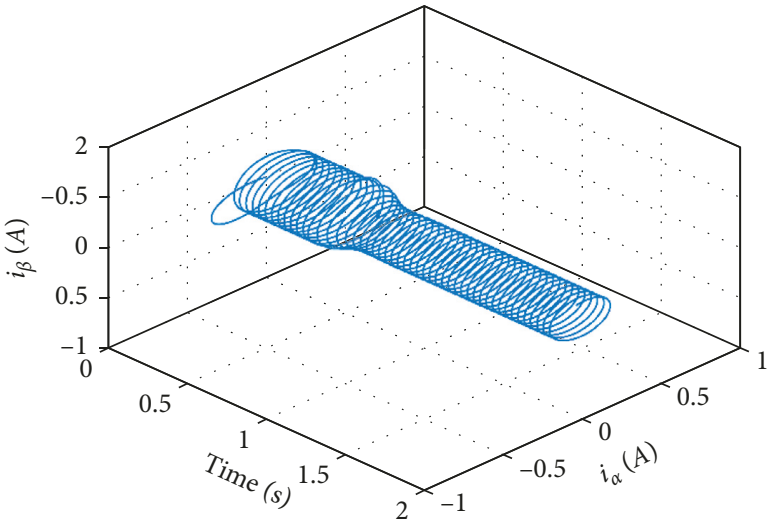

(b)

Figure 10: Continued. 


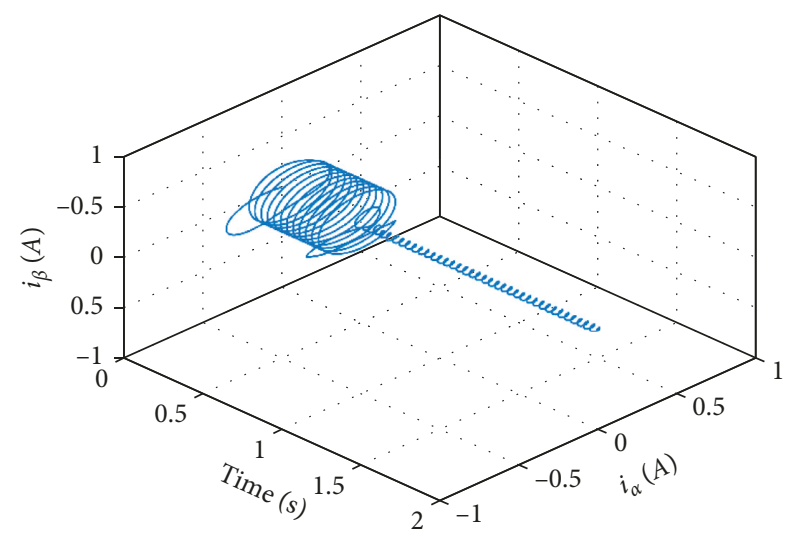

(c)

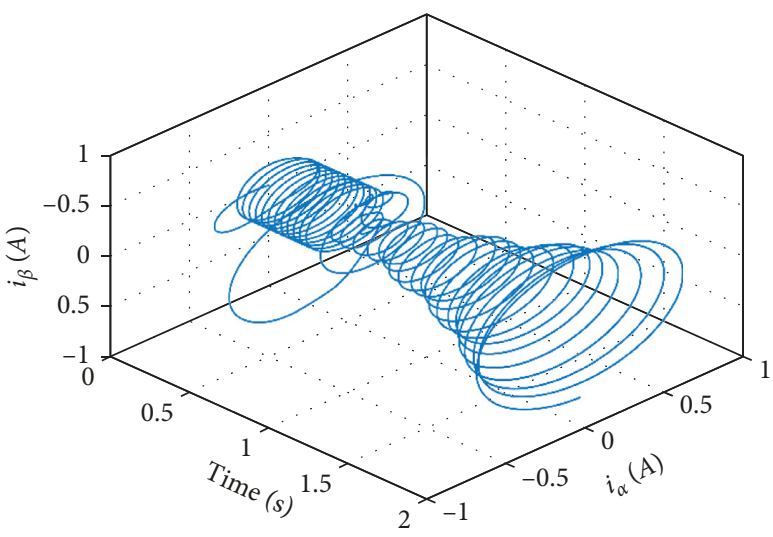

(d)

Figure 10: Simulation results of coil current with the proposed method activated at $t=0.5 \mathrm{~s}$ and the MSFW rotating at the constant speed of $1500 \mathrm{rpm}$. (a) $\kappa=10 ; \theta_{k}=-22^{\circ}$. (b) $\kappa=10 ; \theta_{k}=-85^{\circ}$. (c) $\kappa=100 ; \theta_{k}=-22^{\circ}$. (d) $\kappa=100 ; \theta_{k}=-85^{\circ}$.

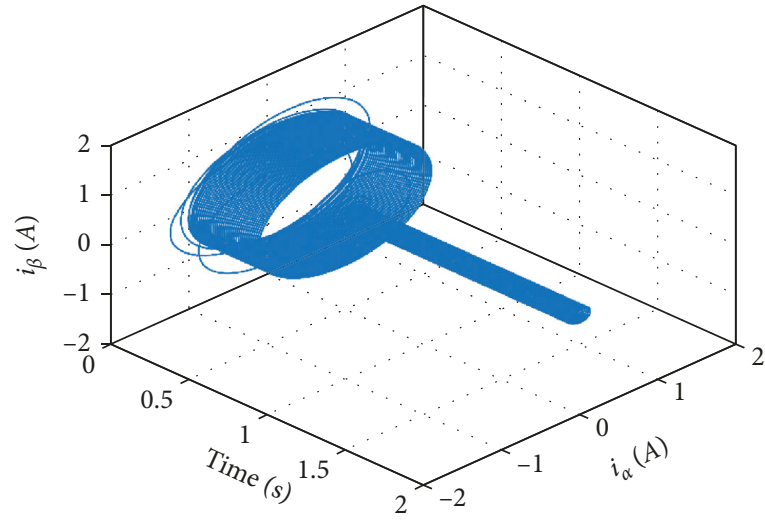

(a)

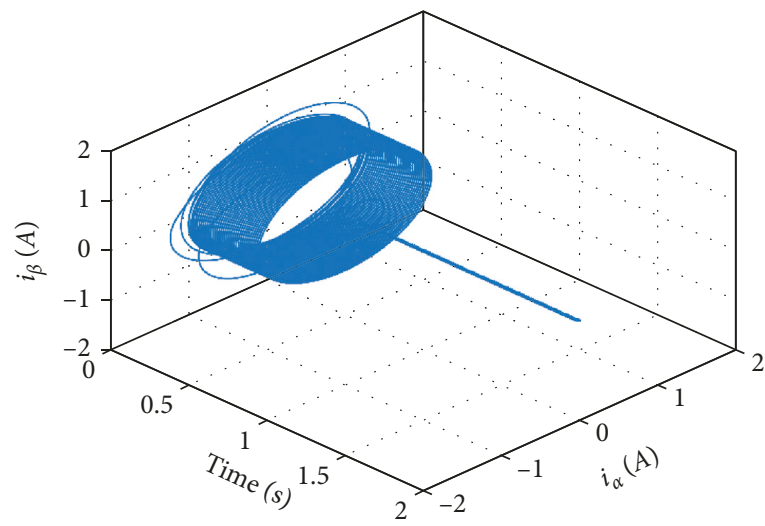

(c)

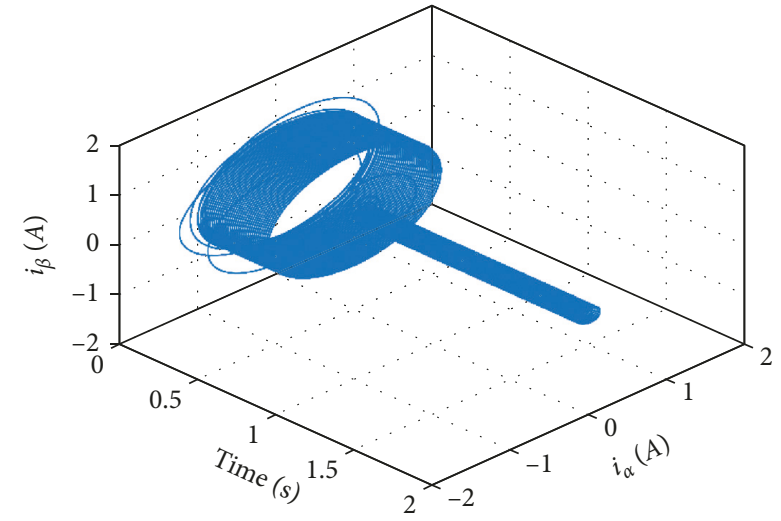

(b)

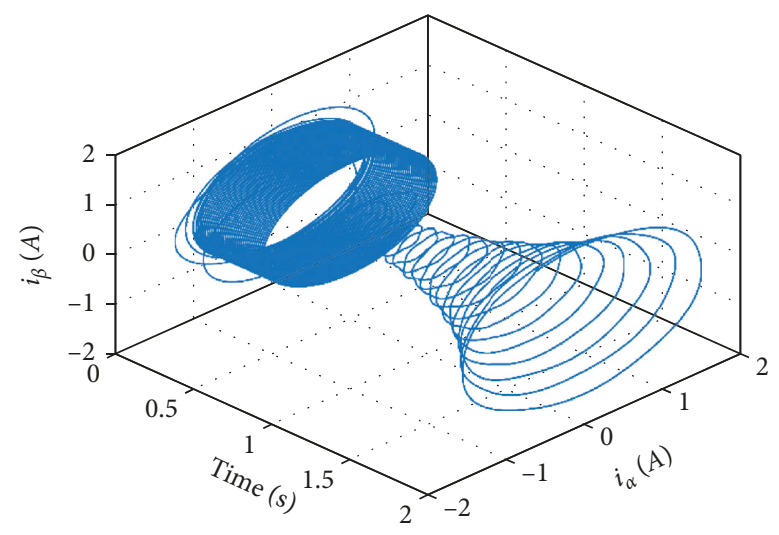

(d)

FIGURE 11: Simulation results of coil current with the proposed method activated at $t=0.5 \mathrm{~s}$ and the MSFW rotating at the constant speed of $4800 \mathrm{rpm}$. (a) $\kappa=10 ; \theta_{k}=-11^{\circ}$. (b) $\kappa=10 ; \theta_{k}=-42^{\circ}$. (c) $\kappa=100 ; \theta_{k}=-11^{\circ}$. (d) $\kappa=100 ; \theta_{k}=-42^{\circ}$.

Furthermore, the vibration torques of the MSFW in the full speed range are measured and presented. The MSFW operates in a speed run-up case from 0 to $5000 \mathrm{r} / \mathrm{min}$. Figure 14 shows the comparative experimental results of the vibration torques without suppression and with the proposed SRF-based synchronous vibration suppression method for $\alpha$ and $\beta$ directions, respectively. As observed from Figure 14(c), the synchronous harmonic increases with the speed running up. The maximum amplitude can reach to $3 \mathrm{Nm}$ at the speed of $5000 \mathrm{rpm}$. Compared to the results 

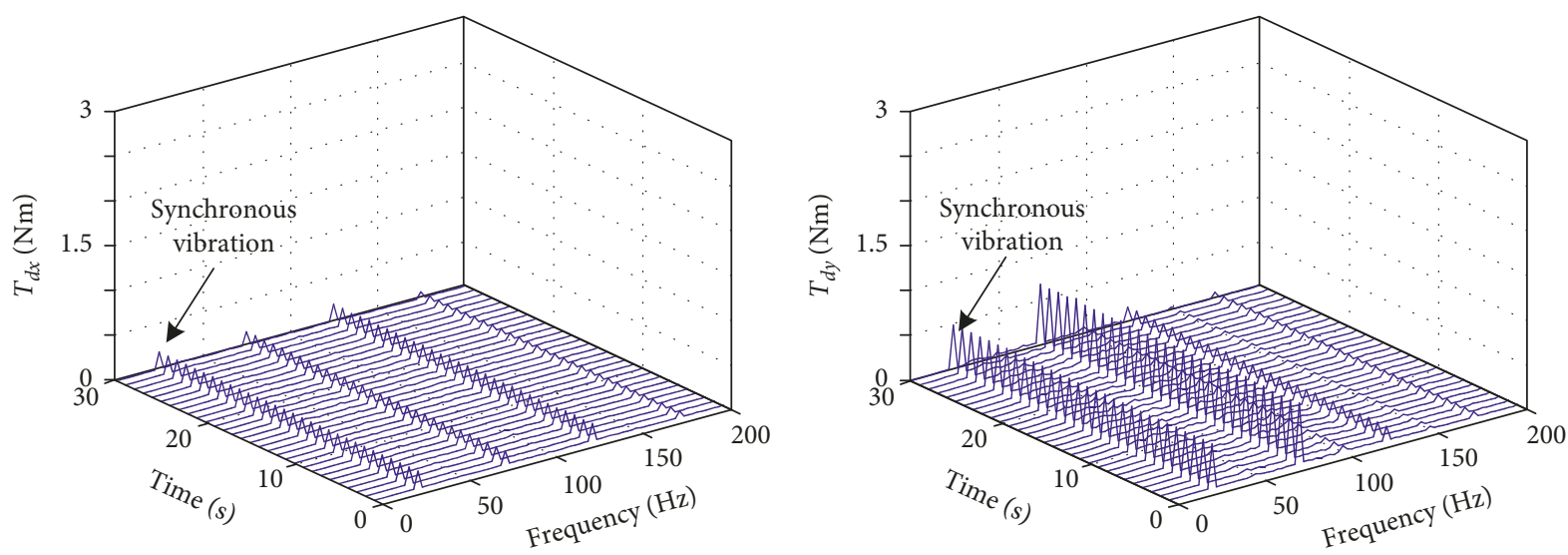

(a)

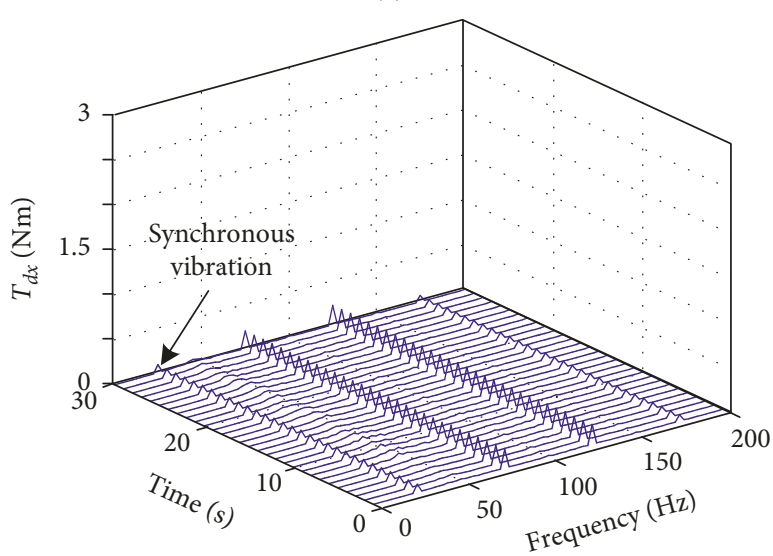

(c)

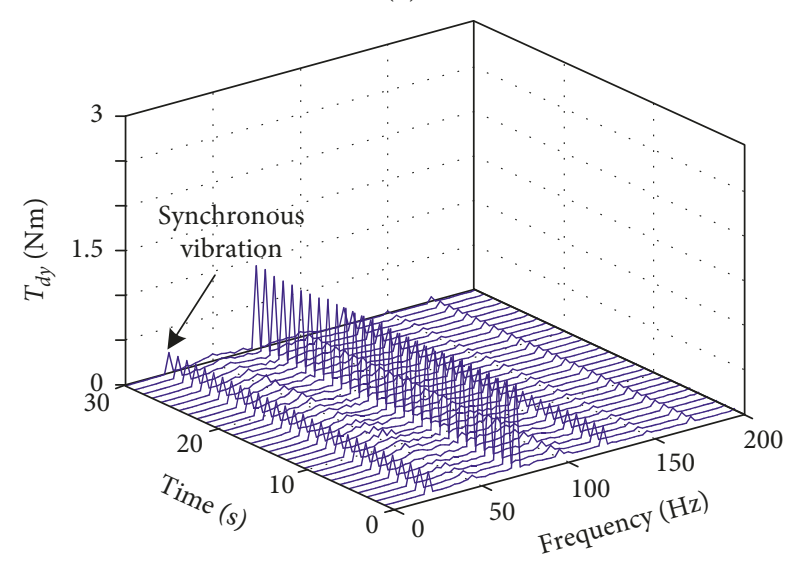

(d)

FIGURE 12: Experimental results for vibration torques measured at the speed of $1500 \mathrm{rpm}$. (a) $T_{d x}$ for $\alpha$ direction without suppression. (b) $T_{d x}$ for $\alpha$ direction with the proposed method suppression. (c) $T_{d y}$ for $\beta$ direction without suppression. (d) $T_{d y}$ for $\beta$ direction with the proposed method suppression.

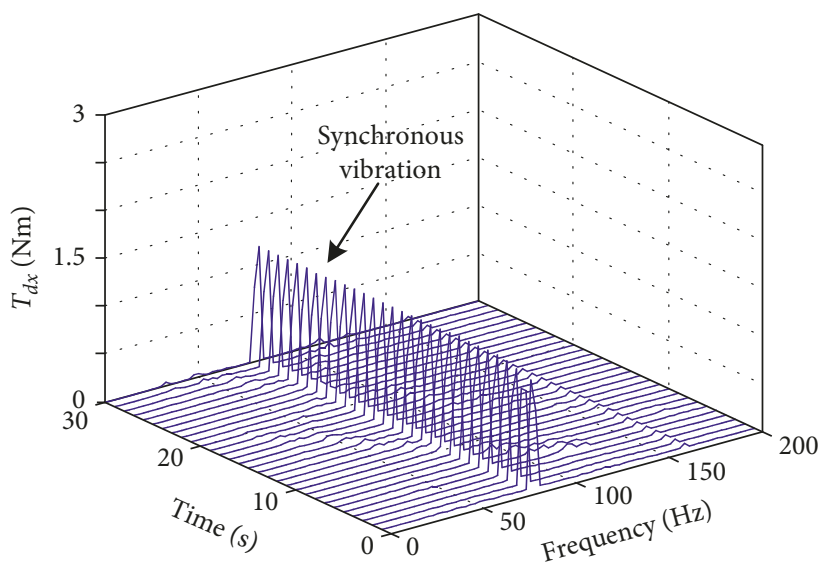

(a)

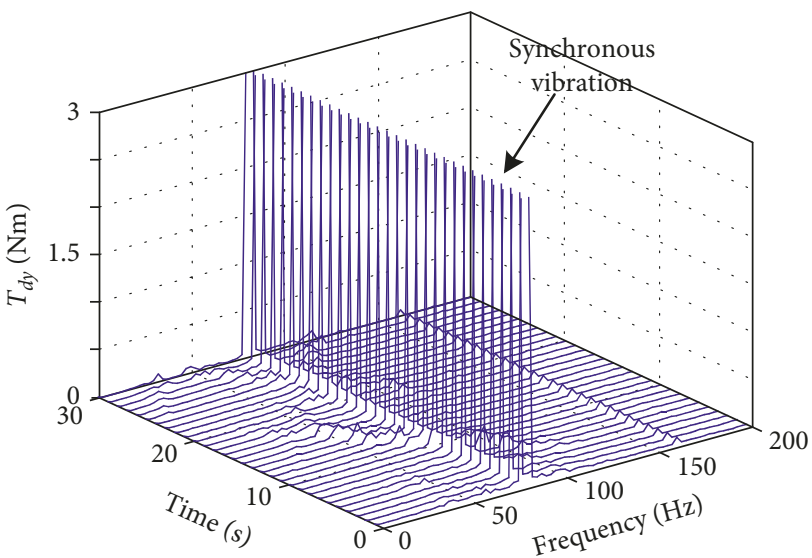

(b)

Figure 13: Continued. 


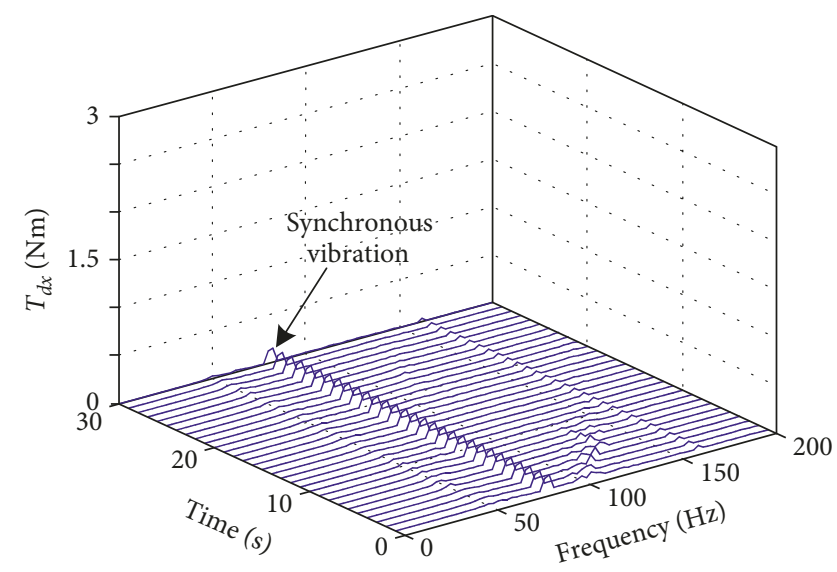

(c)

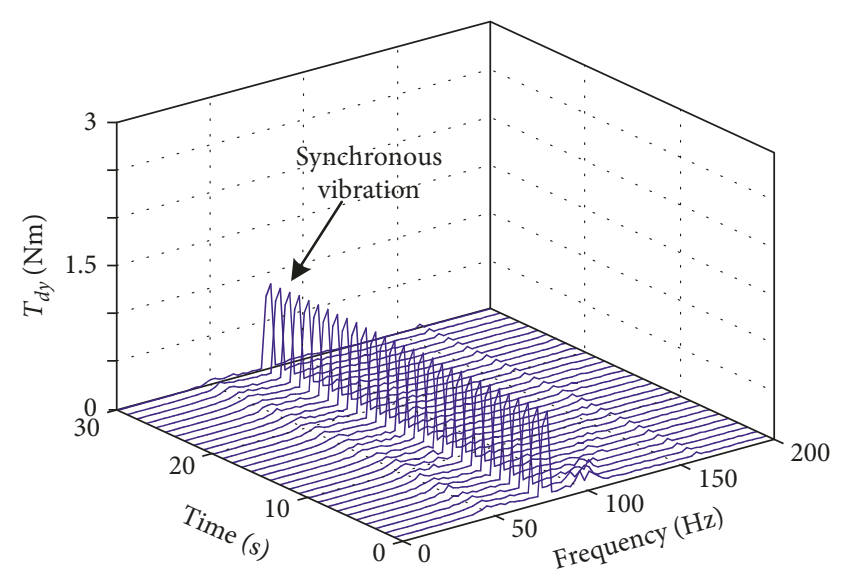

(d)

Figure 13: Experimental results for vibration torques measured at the speed of $4800 \mathrm{rpm}$. (a) $T_{d x}$ for $\alpha$ direction without suppression. (b) $T_{d x}$ for $\alpha$ direction with the proposed method suppression. (c) $T_{d y}$ for $\beta$ direction without suppression. (d) $T_{d y}$ for $\beta$ direction with the proposed method suppression.

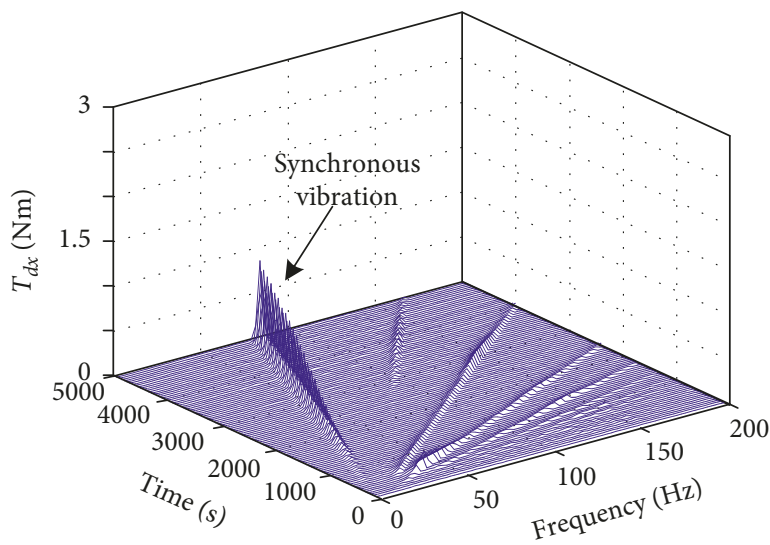

(a)

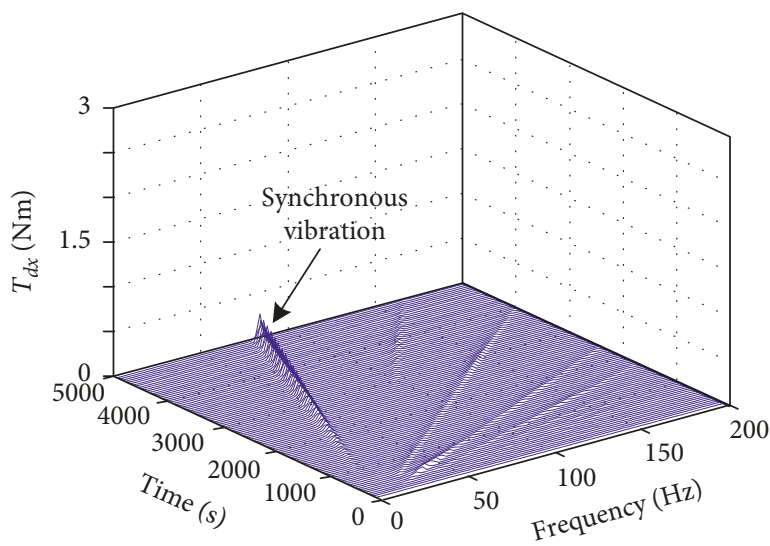

(c)

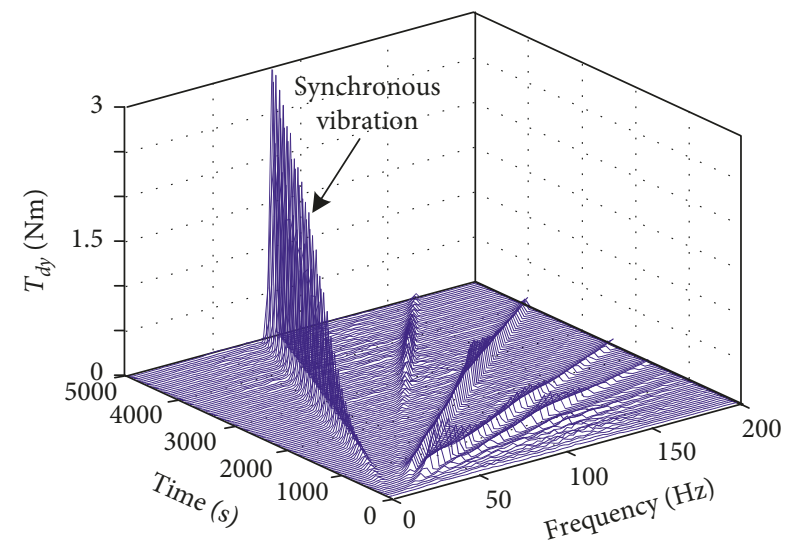

(b)

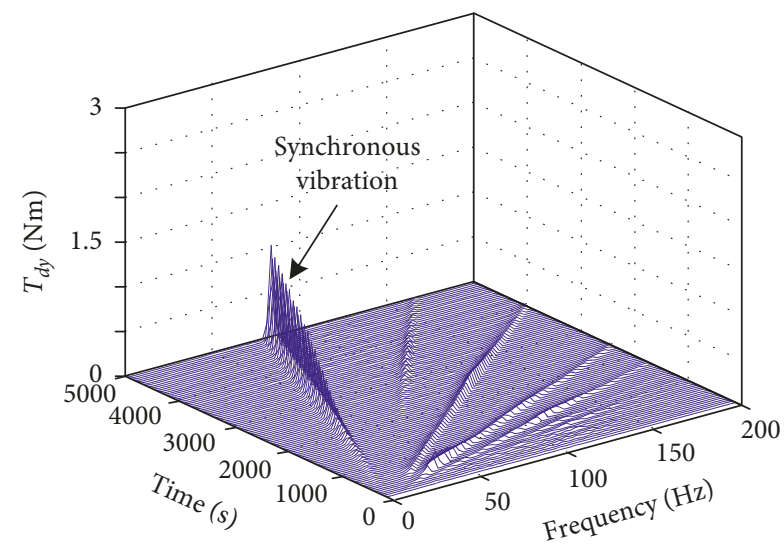

(d)

FIGURE 14: Experimental results for vibration torques measured in a speed run-up condition. (a) $T_{d x}$ for $\alpha$ direction without suppression. (b) $T_{d x}$ for $\alpha$ direction with the proposed method suppression. (c) $T_{d y}$ for $\beta$ direction without suppression. (d) $T_{d y}$ for $\beta$ direction with the proposed method suppression.

without suppression, the synchronous harmonic decreases to a small amplitude with the proposed suppression scheme. As for the synchronous component torque, the maximum amplitude is about $30 \%$ of that without suppression as shown in Figure 14(d). It demonstrates the effectiveness of the proposed method in suppressing the synchronous 
vibration torques. Moreover, the stability of the MIMO coupled system in a speed variation condition is preserved in a good performance.

\section{Conclusion}

In this paper, an improved SRF-based synchronous vibration torque suppression method for MSFW in the presence of gyroscopic effects and coupled dynamics is proposed. Compared to the conventional autobalancing control methods, the proposed method can not only eliminate the synchronous component in the coupled control axes but also preserve the closed-loop stability of the MSFW system by only adjusting the compensation phase. Moreover, the SRFbased vibration control algorithm suppresses the vibration torques significantly with simplified design procedure and low burden for digital signal processing. Simulation and experimental results show that the application of SRF can reduce the synchronous component in coil currents within a wide range of speed. At the constant operating speed, specifically, the synchronous vibration torque is decreased by $70 \%$. In the future work, the SRF method can be extended to the multiple harmonics suppression for the magnetic bearing system.

\section{Data Availability}

The data used to support the findings of this study are available from the corresponding author.

\section{Disclosure}

This work is an improvement on the basis of the conference paper presented in 2018 37th Chinese Control Conference.

\section{Conflicts of Interest}

The authors declare that there are no conflicts of interest regarding the publication of this paper.

\section{Acknowledgments}

This work was supported by the National Natural Science Foundation of China under grant no. 61703203 and Natural Science Foundation of Jiangsu Province under grant no. BK20170812.

\section{References}

[1] R. Siva Srinivas, R. Tiwari, and C. Kannababu, "Application of active magnetic bearings in flexible rotordynamic systems-a state-of-the-art review," Mechanical Systems and Signal Processing, vol. 106, pp. 537-572, 2018.

[2] X. Dai, Z. Shen, and H. Wei, "On the vibration of rotorbearing system with squeeze film damper in an energy storage flywheel," International Journal of Mechanical Sciences, vol. 43, no. 11, pp. 2525-2540, 2001.

[3] C. Peng, J. Sun, X. Song, and J. Fang, "Frequency-varying current harmonics for active magnetic bearing via multiple resonant controllers," IEEE Transactions on Industrial Electronics, vol. 64, no. 1, pp. 517-526, 2017.
[4] X. Qiao and G. Hu, "Active control for multimode unbalanced vibration of flexible spindle rotor system with active magnetic bearing," Shock and Vibration, vol. 2017, Article ID 9706493, 9 pages, 2017.

[5] B. Han, Q. Xu, and Q. Yuan, "Multi-objective optimization of a combined radial-axial magnetic bearing for magnetically suspended compressor," IEEE Transactions on Industrial Electronics, vol. 63, no. 4, pp. 2284-2293, 2016.

[6] G. Schweitzer and E. H. Maslen, Magnetic Bearings: Theory, Design, and Application to Rotating Machinery, Springer, Berlin, Germany, 2009.

[7] J. D. Lindlau and C. R. Knospe, "Feedback linearization of an active magnetic bearing with voltage control," IEEE Transactions on Control Systems Technology, vol. 10, no. 1, pp. 21-31, 2002.

[8] S. Mukoyama, K. Nakao, H. Sakamoto et al., "Development of superconducting magnetic bearing for $300 \mathrm{~kW}$ flywheel energy storage system," IEEE Transactions on Applied Superconductivity, vol. 27, no. 4, pp. 1-4, 2017.

[9] M. Komori, K. Hara, K. Asami, and N. Sakai, "Trial of superconducting magnetic bearings applied to high speed turbine rotor," IEEE Transactions on Magnetics, vol. 54, no. 11, pp. 1-4, 2018.

[10] S. Zheng and R. Feng, "Feedforward compensation control of rotor imbalance for high-speed magnetically suspended centrifugal compressors using a novel adaptive notch filter," Journal of Sound and Vibration, vol. 366, pp. 1-14, 2016.

[11] C. Peng, Y. Fan, Z. Huang, B. Han, and J. Fang, "Frequencyvarying synchronous micro-vibration suppression for a MSFW with application of small-gain theorem," Mechanical Systems and Signal Processing, vol. 82, pp. 432-447, 2017.

[12] J. Zhou, S. Zheng, B. Han, and J. Fang, "Effects of notch filters on imbalance rejection with heteropolar and homopolar magnetic bearings in a $30-\mathrm{kW} 60,000-\mathrm{r} / \mathrm{min}$ motor," IEEE Transactions on Industrial Electronics, vol. 64, no. 10, pp. 8033-8041, 2017.

[13] J. Fang, Y. Ren, and Y. Fan, "Nutation and precession stability criterion of magnetically suspended rigid rotors with gyroscopic effects based on positive and negative frequency characteristics," IEEE Transactions on Industrial Electronics, vol. 61, no. 4, pp. 2003-2014, 2014.

[14] J. Kejian, Z. Changsheng, and T. Ming, "A uniform control method for imbalance compensation and automation balancing in active magnetic bearing-rotor system," Journal of Dynamic Systems, Measurement, and Control-Transactions of the ASME, vol. 134, no. 2, article 021006, 2012.

[15] R. Herzog, P. Buhler, C. Gahler, and R. Larsonneur, "Unbalance compensation using generalized notch filters in the multivariable feedback of magnetic bearings," IEEE Transactions on Control Systems Technology, vol. 4, no. 5, pp. 580-586, 1996.

[16] C. Peng, S. Zheng, Z. Huang, and X. Zhou, "Complete synchronous vibration suppression for a variable-speed magnetically suspended flywheel using phase lead compensation," IEEE Transactions on Industrial Electronics, vol. 65, no. 7, pp. 5837-5846, 2018.

[17] B. Shafai, S. Beale, P. Larocca, and E. Cusson, "Magnetic bearing control systems and adaptive forced balancing," IEEE Control Systems Magazine, vol. 14, no. 2, pp. 4-13, 1994.

[18] J. Kejian, Z. Changsheng, and C. Liangliang, "Unbalance compensation by recursive seeking unbalance mass position in active magnetic bearing-rotor system," IEEE Transactions on Industrial Electronics, vol. 62, no. 9, pp. 5655-5664, 2015. 
[19] C. Bi, D. Wu, Q. Jiang, and Z. Liu, "Automatic learning control for unbalance compensation in active magnetic bearings," IEEE Transactions on Magnetics, vol. 41, no. 7, pp. 2270-2280, 2005.

[20] F. Matsummura, T. Namerikawa, K. Hagiwara, and M. Fujita, "Application of gain scheduled $\mathrm{H}$ infinity robust controllers to a magnetic bearing," IEEE Transactions on Control Systems and Technology, vol. 4, no. 5, pp. 484-493, 1996.

[21] M. Xiang and T. Wei, "Autobalancing of high-speed rotors suspended by magnetic bearings using LMS adaptive feedforward compensation," Journal of Vibration and Control, vol. 20, no. 9, pp. 1428-1436, 2014.

[22] P. Cui, D. Han, G. Zhang, Z. Liu, and B. Han, "Robust odd repetitive controller for magnetically suspended rotor system," IEEE Transactions on Industrial Electronics, vol. 66, no. 3, pp. 2025-2033, 2019.

[23] C. Peng, J. Sun, C. Miao, and J. Fang, "A novel cross-feedback notch filter for synchronous vibration suppression of an MSFW with significant gyroscopic effects," IEEE Transactions on Industrial Electronics, vol. 64, no. 9, pp. 7181-7190, 2017.

[24] F. Gonzalez-Espin, E. Figueres, and G. Garcera, "An adaptive synchronous-reference-frame phase-locked loop for power quality improvement in a polluted utility grid," IEEE Transactions on Industrial Electronics, vol. 59, no. 6, pp. 2718-2731, 2012.

[25] A. Viji and T. A. A. Victoire, "Enhanced PLL based SRF control method for UPQC with fault protection under unbalanced load conditions," International Journal of Electrical Power and Energy Systems, vol. 58, pp. 319-328, 2014.

[26] H. E. P. de Souza, F. Bradaschia, F. A. S. Neves, M. C. Cavalcanti, G. M. S. Azevedo, and J. P. de Arruda, "A method for extracting the fundamental-frequency positivesequence voltage vector based on simple mathematical transformations," IEEE Transactions on Industrial Electronics, vol. 56, no. 5, pp. 1539-1547, 2009.

[27] M. Liserre, R. Teodorescu, and F. Blaabjerg, "Multiple harmonics control for three-phase grid converter systems with the use of PI-RES current controller in a rotating frame," IEEE Transactions on Power Electronics, vol. 21, no. 3, pp. 836-841, 2006.

[28] Y. Han, X. Fang, P. Yang, C. Wang, L. Xu, and J. M. Guerrero, "Stability analysis of digital-controlled single-phase inverter with synchronous reference frame voltage control," IEEE Transactions on Power Electronics, vol. 33, no. 7, pp. 63336350, 2018.

[29] R. Kabiri, D. G. Holmes, and B. P. McGrath, "Control of active and reactive power ripple to mitigate unbalanced grid voltages," IEEE Transactions on Industry Applications, vol. 52, no. 2, pp. 1660-1668, 2016.

[30] K. Cai, C. Peng, Z. Deng, and K. Li, "Vibration suppression control using SRF for MSFW with gyroscopic effects," in Proceedings of the 37th Chinese Control Conference, pp. 3579-3584, Wuhan, China, July 2018.

[31] J. Fang, J. Sun, H. Liu, and J. Tang, "A novel 3-DOF axial hybrid magnetic bearing," IEEE Transactions on Magnetics, vol. 46, no. 12, pp. 4034-4045, 2010.

[32] C. Peng, J. Fang, and P. Cui, "Dynamics modeling and measurement of the microvibrations for a magnetically suspended flywheel," IEEE Transactions on Instrumentation and Measurement, vol. 64, no. 12, pp. 3239-3252, 2015. 


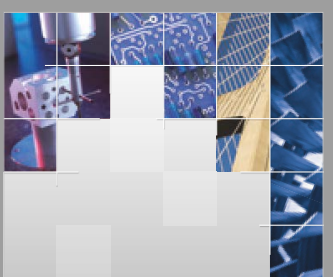

\section{Enfincering}
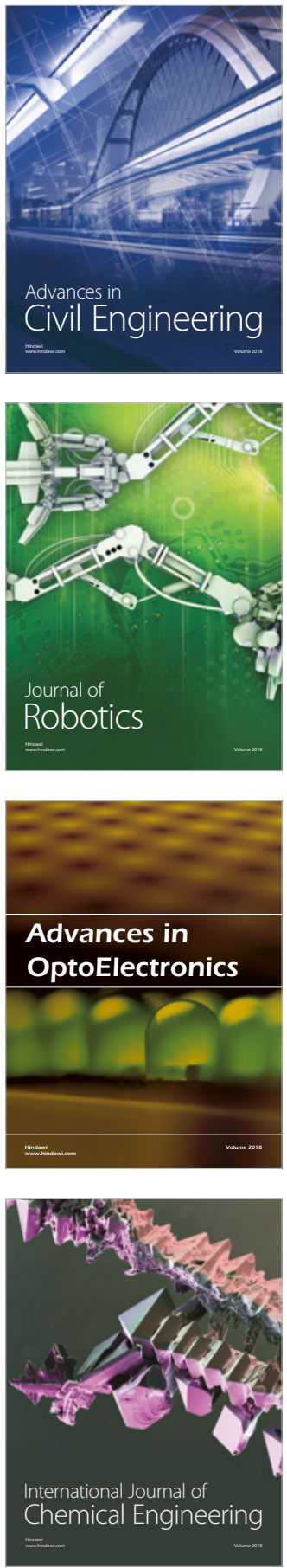

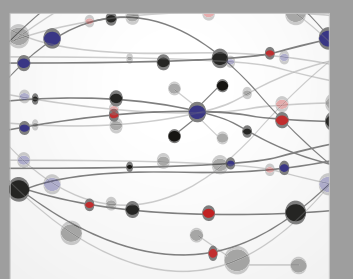

\section{Rotating \\ Machinery}

The Scientific World Journal

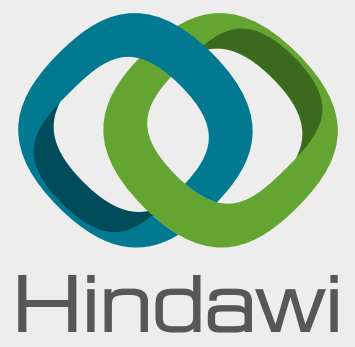

Submit your manuscripts at

www.hindawi.com
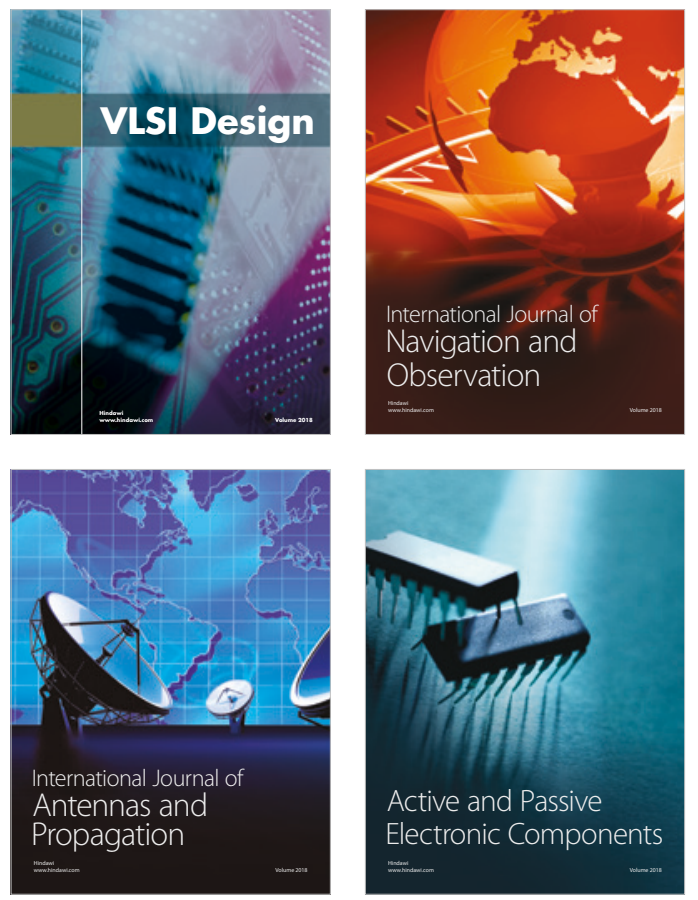
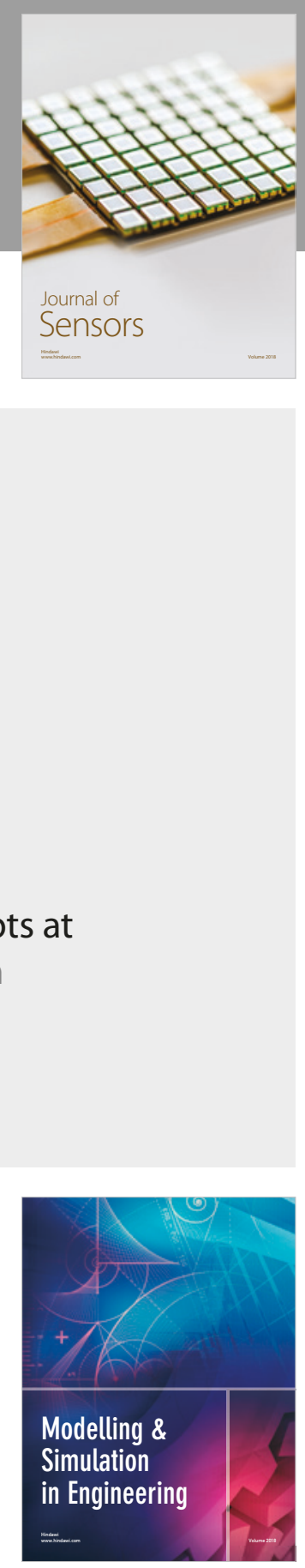

\section{Advances \\ Multimedia}
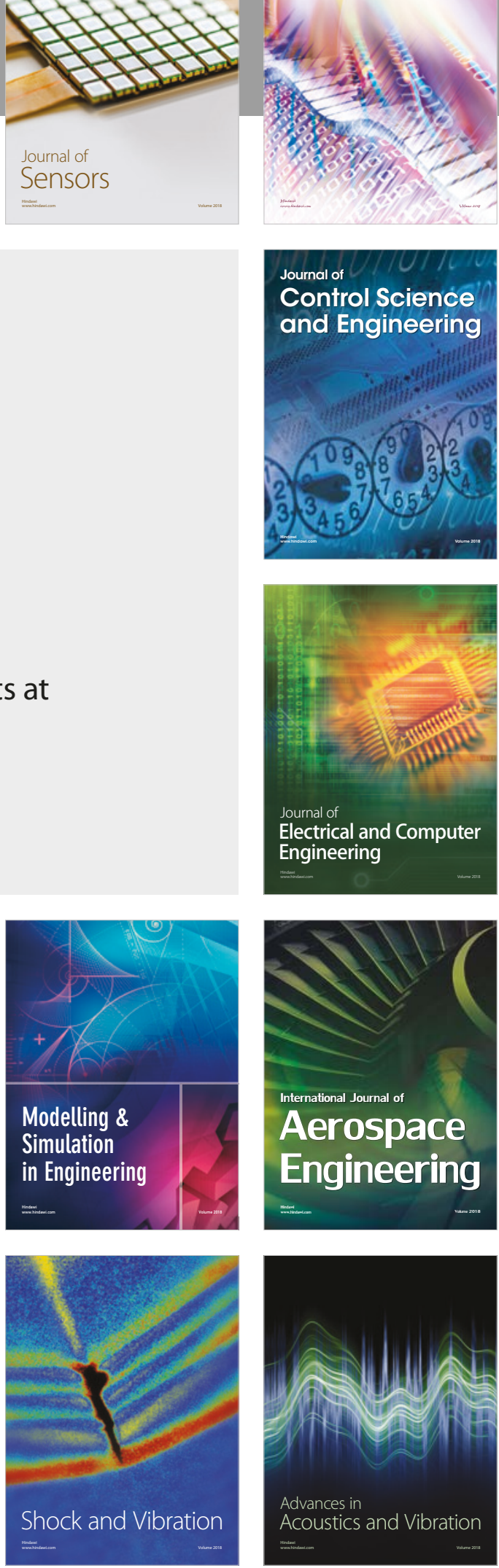